\title{
Las comunidades de práctica en un contexto de gerencia del conocimiento: Estudio de un caso*
}

\author{
Pirela Morillo, Johann** \\ Ocando Medina, Jenny ${ }^{* * *}$ \\ Rincón, Elita****
}

\section{Resumen}

Se presenta una investigación que da origen a la formulación de una propuesta para organizar comunidades de práctica en la Escuela de Bibliotecología y Archivología de la Universidad del Zulia. Se fundamenta a partir de una metodología que incluye el análisis conceptual, epistemológico y empírico. Se efectuó una revisión de varios autores y se adelantó un estudio empírico que partió de la revisión de planteamientos expuestos por la Comisión Organizadora de las Jornadas de Transformación en LUZ y los resultados de un acercamiento a la realidad que se orientó hacia la detección de signos de crisis presentes en la dinámica académico-administrativa de la Escuela de Bibliotecología y Archivología de la Universidad del Zulia, para lo cual se utilizó un cuestionario que posibilitó categorizar las áreas problemas identificadas por los actores-informantes. Luego, se revisan las líneas de desarrollo académico en el área de bibliotecología y ciencias de la información existentes en universidades latinoamericanas y españolas, para concluir que la mencionada Escuela debe concebir y desarrollar un nuevo modelo organizativo, centrado en compartir el conocimiento, mediante comunidades de práctica y para lograrlo deben conformarse tres comunidades: Análisis documental y sistemas de representación del conocimiento, gerencia de la información y el conocimiento y estudios métricos de la información científica y tecnológica.

Palabras clave: Comunidades de práctica, gerencia del conocimiento, gestión educativa, Universidad del Zulia.

Recibido: 02-10-15 . Aceptado: 03-02-28

* Este trabajo es producto de una investigación realizada en el Seminario: Gerencia del Conocimiento, dictado por el Dr. Eustoquio García. Doctorado en Ciencias Humanas. LUZ.

** Profesor de la Escuela de Bibliotecología y Archivología de LUZ. E-mail: jpirela@luz.ve

*** Profesora de la Escuela de Educación de LUZ. E-mail: santora@starmedia.com

**** Profesora de la Escuela de Economía de LUZ. 


\section{Practice Communities in the Context of Knowledge Management: A Case Study}

\section{Abstract}

The formulation of a proposal to organize practice communities in the Library Sciences School at the University of Zulia is presented in this paper. It is based on a methodology that includes conceptual, epistemological, and empirical analysis. A bibliographical review is undertaken of various authors and an empirical study based on a review of the proposals made by the Organizational Commission for the Work Session on Transformation in LUZ is undertaken. The results offer a focus as to the reality of the situation, and signs of a crisis in the academic-administrative dynamic in the Library Sciences School are detected. A questionnaire was utilized to categorize the problem areas identified by the actor-informants. A review of the academic developmental focuses and areas and information available on Latin American and Spanish Universities was undertaken. In conclusion the School of Library Sciences must conceive and develop a new organizational model, centered on sharing knowt edge through practice communities, and to achieve this three communities must be organized: docur mentary analysis and knowledge representation systems, management of information and knowt edge, and metric studies of scientific and technological information.

Palabras clave: Practice communities, management of knowledge, educational management, University of Zulia.

\section{Introducción}

Este trabajo es el producto de una investigación realizada en el Seminario sobre Gerencia del Conocimiento, dictado en el Doctorado en Ciencias Humanas de LUZ. El propósito del trabajo es articular los principios y criterios del enfoque de comunidades de práctica $(C$ de $\mathrm{P})$, con el actual proceso de revisión académico-administrativa que realiza LUZ en general y la Escuela de Bibliotecología y Archivología de LUZ (EBA-LUZ) en particular. El resultado, la aplicación y conformación de comunidades de práctica como forma de organizar y compartir el conocimiento entre la EBA-LUZ, sus egresados ubicados en diferentes sectores y otros profesionales, docentes e investigadores de la bibliotecología, archi- vología y ciencias de la información de América Latina y España.

Para el desarrollo de la propuesta, se efectúa una aproximación conceptual y epistemológica para fundamentar la idea de comunidad de práctica en el contexto de lo que se denomina en estos momentos gerencia del conocimiento. Se puntualiza sobre las definiciones de Brown (2000) y Mc. Dermott (2000). En este sentido, se observa coincidencia entre los autores, al afirmar que una comunidad de práctica se define como un grupo de personas que se comunican cara a cara o por vía electrónica y comparten informaciones y experiencias.

Luego, se precisan las funciones y características de las comunidades de práctica y se abre una interrogante sobre la posibilidad de introducir este enfoque 
en el ámbito universitario. Para desarrollar este punto, se señalan algunos factores que pueden obstaculizar la implementación del enfoque en las organizaciones universitarias.

Posterior a la definición de las estrategias medulares que orientan la implementación de las comunidades de práctica en las instituciones universitarias, se realiza un acercamiento empírico a la realidad organizacional de la EBA-LUZ, para contrastar los planteamientos teórico-conceptuales con la realidad. A partir de este proceso, se detectan signos de crisis que ameritan la aplicación del enfoque de comunidades de práctica, como una alternativa de la organización para producir y compartir el conocimiento.

En la EBA-LUZ se ha asumido un proceso de revisión estructural, que pasa por reflexionar sobre diferentes aspectos que tocan la dinámica académico-administrativa. En este sentido, luego de la reorganización, la construcción colectiva de una filosofía de gestión, la revisión de tendencias en materia de líneas y áreas prioritarias en bibliotecología, archivología y ciencias de la información en Latinoamérica y España; se concluye que las comunidades de práctica que la Escuela debe conformar y estructurar son: Análisis documental y sistemas de representación del conocimiento, gerencia de la información y el conocimiento y estudios métricos de la información científica y tecnológica.

Se considera que este enfoque permitirá no sólo compartir el conocimiento que se genera como resultado del quehacer académico, sino repensar la estructura organizacional de la Escuela en el marco de las transformaciones que se le plantean a la Universidad del Zulia.

\section{Hacia una definición de Comunidades de Práctica}

Empecemos por ubicar los términos comunidad y práctica, de acuerdo con el Diccionario de la Real Academia Española de la Lengua (DRAE, 1992). En cuanto a Comunidad (comunitas), se expresa que es la Calidad de común, de lo que, no siendo privativamente, pertenece o se extiende a varios.

Pasquali (1998), al referirse al carácter comunicante de la ciudad, señala que todas las grandes utopías de la humanidad fueron utopías -de-la-relaciónhumana que partieron de una cohabitación perfecta en el espacio como apófansis o externalización de un "con-vivir", como ratio essendi et cognoscendi del paraíso político. En sus prístinos étimos griegos, los conceptos de comunidad y comunicación tienen connotaciones que se vinculan con lo colectivo. El mundo griego entendió que la comunidad hace referencia a un topos (lugar), el cual se organiza y articula gracias a la comunicación. En el mundo griego, la comunidad es la koinonía.

Esta explicación de Pasquali, que como vemos se refiere al carácter de lo comunitario y colectivo, puede ser extrapolada a nuestro caso particular si entendemos que el topos hace referencia al espacio en el cual se dan las relaciones e interacciones entre sujetos que comparten intereses personales y organizacionales comunes: la organización; y la forma de integración colectiva de experiencias y saberes es la koinonia: la comunidad.

En cuanto al vocablo práctica, el DRAE señala que proviene del latín practicus: 1. las facultades que enseñan el 
modo de hacer una cosa. 2. Se incluyen también las nociones de experimentado, versado y diestro en una actividad.

Entonces, una comunidad de práctica $(C$ de $P$ ) en una primera aproximación terminológica hace referencia al reconocimiento de las facultades, conocimientos, experiencias que se comparten en un espacio que no es individual sino común, colectivo: la koinonía, la comunidad: la organización.

Recurriendo ahora a los autores que han propuesto definiciones del concepto de $\mathrm{C}$ de $\mathrm{P}$ en el contexto de la gerencia del conocimiento, como Mc. Dermott (2000), se observa que este tipo de comunidades son conocidas también como comunidades de conocimiento o de aprendizaje. Las C de P son grupos de personas que comparten entre sí información, reflexiones, consejos e ideas, mediante acciones de comunicación frente a frente o por vía electrónica.

En las acciones comunicativas mediadas por computador, el proceso es más complejo, porque el conocimiento que se comparte es redificado, el trabajo deslocalizado y surgen nuevas lógicas discursivas, como producto de la interacción en espacios virtuales, entendidos como nuevos espacios de actuación social, de comunicación interactiva (Caballero, citado por Sánchez, 2000).

Puede observarse que el elemento articulador de la dinámica de las $\mathrm{C}$ de $\mathrm{P}$ es la comunicación, ya sea cara a cara o mediada tecnológicamente.

Brown (2000), define las C de P como un grupo de individuos que han estado trabajando juntos durante un largo período, y que por haber compartido prácticas también comparten ricas expe- riencias. Además, plantea el autor, han elaborado sus propios mecanismos de confianza, porque todos saben lo que cada uno de ellos es capaz de hacer. Eso permite, que dentro de la comunidad las ideas fluyan fácilmente.

En conclusión, la idea de $\mathrm{C}$ de $\mathrm{P}$, conocidas también como comunidades de aprendizaje o conocimiento se apoya teóricamente en el proceso de comunicación humana, entendido como la posibilidad de interacción y reciprocidad, por un lado, y por el otro se apoya en un concepto de aprendizaje y de cognición, en el que subyace la dimensión social del conocimiento (Goldman, 1998).

\section{Enfoques teórico-epistemológicos que sustentan la noción de comunidades de práctica}

Las $\mathrm{C}$ de $\mathrm{P}$ son formas de organización y de actuación colectiva, en un contexto de gerencia del conocimiento. Por ello, estas comunidades también reciben el nombre de comunidades de conocimiento. Asumir el enfoque de Gerencia del Conocimiento (GC) supone ubicarse en una epistemología que reconoce el paso de la razón instrumental a la razón sensible, al incorporar un nuevo tipo de pensamiento: el complejo.

Se trata de entender que el conocimiento, en estos momentos, es más que resultados de procesos investigativos científicos e incluye las experiencias y el know-how. Esta concepción critica el relativismo y determinismo que ha impregnado las concepciones tradicionales de conocimiento, las cuales lo vinculan sólo a lo "científico". Los planteamientos 
emergentes en torno al concepto de conocimiento, proponen aproximarse al mismo, asumiendo la disolución de los límites entre ciencias y pseudociencias y el vaciamiento del significado de objetividad. En este sentido, autores como lan Hacking (1996) proponen que la ciencia es simultáneamente un conocer (teorías científicas) y un intervenir (tecnología). Tales planteamientos ubican la noción de conocimiento desde los denominados estudios sobre ciencia, tecnología y sociedad (CTS).

Paralelo a lo anterior, si se acepta que el conocimiento puede compartirse en comunidades organizacionales conformadas para su dinamización y apalancamiento, se entiende entonces que este proceso plantea además el despliegue de estrategias de interacción dialógica y compleja, derivada de la complejidad intrínseca de las acciones humanas (Wagensberg, 1994). Desde esta perspectiva, se entiende la organización como un sistema vivo (hombre o conjunto de hombres) y abierto, que comparte información interna y externamente, y su evolución es producto de los cambios de tales interacciones. De manera que en la complejidad puede también apoyarse el planteamiento de las $C$ de $P$.

Pasando ahora al plano de lo teóri$\mathrm{co}$, la idea de $\mathrm{C}$ de $\mathrm{P}$ hunde sus raíces en la Teoría de Vigotsky (Wertsch, 1985), según la cual el conocimiento de todo individuo conforma una suerte de núcleo que le es propio y emplea para afrontar sus actividades prácticas, no obstante alrededor de dicho núcleo se sitúa la zona de desarrollo próximo, en donde el sujeto, si bien, tiene algún conocimiento requiere de "ayuda" para abordar lo que se le pre- sente. Esta "ayuda" es el ámbito de conocimientos de otros sujetos. Para que esto se de, se necesita que cada sujeto, inmerso en la dinámica, sea consciente de lo que puede ofrecer a los demás. Debe tenerse presente que el conocimiento visto de esta manera no reside en el individuo, sino entre ellos.

Al perfilar su propuesta por la vía del entrelazamiento del potencial biológico de las personas con las fuerzas culturales, Vigotsky daba cuenta por una parte de la influencia de la filosofía marxista que en su tiempo marcó una nueva dinámica productiva y, por la otra, ponía en evidencia el carácter social del conocimiento.

Otra categoría conceptual que en el contexto de las comunidades de práctica se asume con mucha fuerza es la noción de Cognición contextualizada. En este sentido, autores como Brown, CoIlins y Duiguid (1989), plantean que tanto el aprendizaje como la cognición son procesos fundamentalmente contextualizados, muy alejados de la premisa de los ambientes educativos tradicionales donde se maneja el aprendizaje como un elemento abstracto, sin vinculaciones con el entorno de las personas.

Arcila (2000), siguiendo estas ideas, sostiene que desde la óptica de la cognición contextualizada, las actividades y la cultura son el marco donde se conforman los conceptos, que a su vez, nutren la estructura de conocimientos de los sujetos. El conocimiento se convierte así en una herramienta multipropósito que puede ser empleada en las diversas actividades que se suscitan dentro de una cultura.

Otro concepto en el que se apoya la idea de $C$ de $P$ es el de Gestión del Conocimiento (GC). En este caso, más que 
contribuir a fundamentar la idea de comunidades de práctica, constituye el enfoque desde el cual se puede concretar esta noción y forma de aprendizaje. El concepto de Gestión del Conocimiento, surge formalmente según Arcila (2000:2), en el campo organizacional de Estados Unidos y Europa; donde ésta particular expresión comenzó a formar parte de la jerga de los gerentes.

Pero, para profundizar en el alcance y naturaleza de la Gerencia del Conocimiento (GC), es necesario estudiar los argumentos expuestos por Wah (1999), quien plantea que el foco de la GC consiste en aprovechar y reutilizar los recursos que ya existen en la organización, de modo tal que las personas puedan seleccionar y aplicar el conocimiento producido por los sujetos que integran la organización. Una de las formas de materializar esto, señala la autora, es generando un ambiente donde el aprendizaje sea interactivo y en el que los involucrados puedan transferir y compartir lo que saben, de forma rápida, agregando ese saber a su estructura cognitiva, lo que se traduce en un nuevo conocimiento.

\section{Función y características generales de las comunidades de práctica en el contexto organizacional}

Una vez que se ha precisado conceptual y epistemológicamente la idea de $C$ de $P$, es pertinente analizar sus funciones esenciales.

Las $\mathrm{C}$ de $\mathrm{P}$ desempeñan funciones importantes en una organización. Entre estas funciones están: a) Compartir el conocimiento: que significa compartir ideas y reflexiones tácitas y complejas con el propósito de ayudarse entre sí para resolver problemas y conseguir innovaciones.

b) Poseer y desarrollar capacidades de conocimiento: que implica desarrollar un enfoque común, desarrollar y gerenciar las mejores prácticas y desarrollar la competencia organizacional.

Las $\mathrm{C}$ de $\mathrm{P}$ pueden funcionar a escala local y global. Las comunidades locales realizan reuniones semanales sin orden del día, poseen una biblioteca de datos, cuentan con un miembro coordinador, administran programas de tutorías y realizan procesos de trabajo. Las comunidades globales se estructuran a partir de varias comunidades locales, poseen un coordinador miembro de una red global, se comunican mediante teleconferencias y pueden reunirse varias veces al año con los coordinadores locales.

Para fortalecer las funciones de las comunidades de práctica es importante tener en cuenta:

- El nivel gerencial debe concentrar sus intereses en aspectos relevantes para la productividad y los miembros de la $C$ de $P$.

- Jentro de las $C$ de $P$ debe exisÿir un líder que más que coordinar el trabajo como tal propicie un ambiente que facilite la interrelación de sus miembros.

- Debido a la naturaleza estructural de las $C$ de $P$, debe facilitarse y estimularse a las personas para que puedan hacer sus aportes, sin que por ello desatiendan sus otras labores.

- Teniendo presente que es sumamente complicado cambiar la cultura de 
una organización, es recomendable que el conocimiento generado en las $\mathrm{C}$ de $\mathrm{P}$, se maneje como un aporte al cuerpo de valores que maneja la empresa.

- Se deben generar espacios para que los involucrados puedan analizar situaciones de manera conjunta y también compartan informaciones.

- Facilitar el acceso a las C de P, permitiéndose así la incorporación de aportes y nuevos miembros.

- Establecer un diálogo abierto donde se analicen los asuntos que son de real importancia

Las $\mathrm{C}$ de $\mathrm{P}$ se caracterizan por los siguientes aspectos:

1. El conocimiento compartido requiere o se fundamenta en la confianza y reciprocidad, así como en la red de conexiones y relaciones.

2. Las comunidades se desarrollan a través de etapas naturales.

3. Los miembros de las comunidades necesitan contactos personales (cara a cara, vía electrónica o por teléfono), para desarrollar la confianza necesaria, a fin de compartir los problemas que se presentan y solicitar ayuda a los demás.

4. Los líderes de las comunidades deben ser los miembros más respetados y que posean más elementos de contenido para compartir.

5. Las comunidades organizan y manejan su propia información y conocimiento. Esto refleja la forma de pensar de la comunidad y es importante para la administración de las mejores prácticas.

\section{5. ¿Es posible organizar las universidades en función de conformar comunidades de práctica?}

Aunque la estructura del ambiente universitario generalmente no propicia la implementación de $\mathrm{C}$ de $\mathrm{P}$, no obstante cuando se observan grupos de investigadores reunidos en torno a un tópico particular, con un nivel satisfactorio de cohesión, comunicación y empatía, puede decirse que se trata de una comunidad.

Sin embargo, no cabe duda de que existen diferentes factores que impiden la ejecución de este modelo en las organizaciones universitarias. Estos factores pueden ser:

* Factores históricos que han prevalecido en la construcción del modelo de Universidad en América Latina, y a la manera cómo se entiende la organización y estructuración del conocimiento, todo lo cual responde al esquema universitario europeo, heredero de la tradición medieval (García, citado por De Galicia y Castillo 2000).

* Factores estructurales-organizacionales: Que parten de la fragmentación y concepción desarticulada del conocimiento, junto con modelos organizativos piramidales y burocráticos. Modelos que ya se ven agotados frente a las nuevas condiciones del desarrollo científico y tecnológico y ante las nuevas formas de organización de la producción (DGPU, 1998:15).

* Factores financieros: Derivados de las restricciones financieras y presupuestarias que han afectado la calidad y 
han dificultado las tareas de investigación (UNESCO, 1998).

* Factores políticos: Representados por el agotamiento del patrón tradicional de relación entre los gobiernos, donde los criterios de financiamiento han estado basados más en los patrones de relaciones políticas o de estricto ahorro financiero, que en condiciones de equidad y calidad.

* Factores sociales: Que se observan en la rigidez para adaptarse al patrón emergente de relaciones con la sociedad contemporánea.

* Factores culturales: Asociados al carácter anti-intelectual de la sociedad-cultura venezolana, en cuya estructura privan más elementos de magia y religión, que de reflexión y análisis (Albornoz, 2000). A pesar de estos factores, y recurriendo al espíritu trabajador, cooperativo, creativo y humano de muchos universitarios, se considera posible que en la Universidad se introduzca la lógica organizativa de las comunidades de práctica, para comenzar a compartir y producir conocimiento a escala no sólo local y nacional sino internacional. Esto es posible si los universitarios crean una cultura organizacional centrada en la participación y en la relación humana. En este sentido, se cree que es necesario concebir una estrategia general de implementación que toque la redimensión organizacional y funcional de la institución universitaria.

La posible implementación del modelo de gerencia del conocimiento, y más específicamente del enfoque organizacional que supone las comunidades de práctica, pasa primeramente por la ejecución de las siguientes estrategias medu- lares: Construir un nuevo modelo de universidad compatible con las comunidades de práctica; definir una filosofía de gestión, en la que se declaren, asuman e interioricen valores organizacionales claves para la implementación de las comunidades de práctica; identificar las áreas o líneas temáticas prioritarias que darán organicidad y estructura a las comunidades y conformar las comunidades de práctica.

\section{Estrategias medulares que orientan la implementación del enfoque de comunidades de práctica en el contexto universitario}

El enfoque de las comunidades de práctica como un componente medular de la gerencia del conocimiento, se puede aplicar en el contexto universitario si se parte del redimensionamiento organizacional como estrategia inicial de implementación.

En este sentido, se cree que la primera estrategia medular para implementar el enfoque de comunidades de práctica en el ámbito universitario es repensar la estructura organizativa piramidaljerárquica, organizada por facultades y escuelas e inspirada en el denominado modelo napoleónico, prevaleciente en muchas universidades latinoamericanas. La idea es asumir un modelo matricial departamental que supone relaciones más horizontales entre los miembros de la organización.

Los principios organizacionales básicos que subyacen en el modelo matricial son: la descentralización integral de responsabilidades homogéneas; diseños 
organizacionales orientados a clientes; minimización de regulaciones centralistas y estímulos a los emprendimientos; desconcentración de lo operativo y estratégico puntual; gestión personalizada, centrada en indicadores de calidad; inducción sinérgica, cultura de efectividad; organización para aprendizaje permanente; adopción de modelos tendentes a la calidad; conservación de los controles estratégicos.

Se cree que un modelo organizativo departamentalizado y descentralizado es el más adecuado para la conformación de comunidades de práctica, ya que como se dijo antes, esta forma de organización supone relación, interacción y reciprocidad, características que se asocian más con las estructuras matriciales y horizontales como las que plantea el modelo departamental.

La segunda estrategia medular de implementación es definir y construir colectivamente una filosofía de gestión en la que se declaren, asuman e interioricen valores organizacionales claves para la implementación de las comunidades de práctica. En este sentido, se considera estratégico abrir canales de participación de todos los actores involucrados con la dinámica organizacional. Involucrase en la participación significa un involucramien-

to mental y emocional, esto es, se involucra el yo del individuo y no sólo sus habilidades (Davis y Newstron, 1991).

Además de la participación, como principio articulador del proceso de construcción de una filosofía de gestión compartida, es fundamental asumir algunos criterios generales, como por ejemplo el compromiso de todos los actores con la visión, para lo cual se requiere una construcción cuidadosa del camino a seguir y una comunicación fluida y eficaz. Otro criterio por considerar es la capacidad generativa de la organización en propiciar una plataforma para la creación de ideas y acciones que permitan tomar decisiones sobre el mejor uso que se le puede dar al conocimiento.

La tercera estrategia medular de implementación de las $\mathrm{C}$ de $\mathrm{P}$ es identificar las áreas o líneas temáticas prioritarias que darán organicidad y estructura a las comunidades. Este aspecto es de suma importancia, ya que se trata de definir los temas donde girarán las $\mathrm{C}$ de $\mathrm{P}$, las cuales tendrán una conformación no sólo organizacional-local, sino nacional e internacional. Por ello, se propone revisar las tendencias que en cada disciplina se están trabajando con interés prioritario e identificar tendencias, para conformar y organizar las comunidades.

En vista de que los miembros de las comunidades necesitan contacto frecuente, las comunicaciones y encuentros pueden ser de tipo cara a cara o por vía electrónica. En la conformación de las comunidades no debe olvidarse que es necesario nombrar un líder, que debe ser el miembro más respetado de la comunidad científica. En líneas generales cuando se organizan las comunidades es necesario tomar en cuenta las siguientes preguntas: ¿En qué va a centrar su atención?; ¿Cuáles van a ser los temas de interés?; ¿Cuáles van a ser sus límites?; ¿Cuáles son los fundamentos para la acción?; ¿Cuál es el esquema de valores sobre el que se va estructurar?; ¿Se va a construir un sitio en Internet sobre la comunidad?; 
¿Se piensa tener un coordinador cuya tarea sea la de mantener unida a la comunidad?

Como las $\mathrm{C}$ de $\mathrm{P}$ tienen el propósito de desarrollar las capacidades de sus miembros y la creación e intercambio del conocimiento, su funcionamiento es muy variado, desde los que se reúnen periódicamente en una comida de trabajo, a otros que trabajan a través del e-mail.

Algunas tienen agendas específicas y otras apenas tienen agenda; unas están estructuradas formalmente y otras, informalmente.

\section{Aplicación de las estrategias medulares del enfoque de comunidades de práctica a la EBA-LUZ}

En este punto se desarrolla una propuesta, relacionada con la implementación del enfoque de comunidades de práctica en la Escuela de Bibliotecología y Archivología de la Universidad del Zulia (EBA-LUZ). La implementación del enfoque, además de considerar el análisis conceptual y epistemológico, partió de realizar una investigación empírica, para caracterizar la dinámica académico-administrativa de la EBA-LUZ y contrastarla con los planteamientos teórico-conceptuales de las $\mathrm{C}$ de $\mathrm{P}$. Los resultados de este acercamiento empírico justificaron y evidenciaron la necesidad de que la EBA-LUZ conciba y desarrolle un modelo organizativo que privilegie la producción y comunicación colectiva del conocimiento que se genera como producto de su práctica académica.
Igualmente, conviene señalar que la EBA-LUZ ha desarrollado alguna de las estrategias del enfoque de $\mathrm{C}$ de $\mathrm{P}$, específicamente las dos primeras que se proponen, sobre la base de incorporar los lineamientos propuestos por la Comisión para la Transformación de LUZ (s/f), los cuales apuntan hacia la reorganización académico-administrativa de la Universidad. Se busca generar un salto cualitativo del modelo vertical-piramidal al horizontal-matricial.

La Universidad del Zulia, entonces, se ha planteado la posibilidad de asumir un nuevo modelo, centrado en la departamentalización, entendida como el agrupamiento de recursos organizacionales, mediante un acto administrativo decidido corporativamente, con la finalidad de obtener mejores resultados conjuntos y destinados a lograr, en diferentes puntos de la estructura organizacional, la confluencia de tareas a través del modelo Departamento, para la ejecución de contenidos afines, delimitados por responsabilidades específicas, objetivos y metas puntuales, con suficientes recursos de autoridad, decisión y logística, en correlación concertada de esfuerzo hacia un fin determinado apoyado en procesos de gestión, coordinación intra y extrainstitucional, formulación, ejecución, medición, información, educación, control y desarrollo de planes alineados e integrados al ámbito general de la corporación matriz (Figueras, citado por la Comisión para la Transformación de LUZ, 2001).

Las estrategias de implementación del enfoque de $C$ de $P$ en la EBA-LUZ se enmarcan en este contexto de transformación. 


\subsection{Acercamiento empírico: Detectando signos de crisis, descubriendo una necesidad}

El acercamiento empírico consistió en realizar una investigación diagnóstica para identificar los signos de crisis o las áreas problemas prioritarias que están presentes en la dinámica académico-administrativa de la EBA-LUZ. Siguiendo una lógica inductiva, centrada en el reconocimiento de incidentes que se observan en la realidad, se diseñó un cuestionario con dos preguntas abiertas, cuya finalidad fue explorar en los informantes (estudiantes y egresados) los problemas esenciales que a juicio de ellos debían ser atendidos con mayor prioridad y las soluciones que pueden aportarse para resolver tales problemas.

El propósito de recabar esta información consistió también en la construcción de una agenda de trabajo que agruparía los puntos esenciales que se discutieron en unas jornadas de evaluación de la EBA-LUZ, la cual contó con la participación de profesores, estudiantes y egresados. Para alcanzar tal fin, se convocó a una asamblea abierta, en la que se expresó la necesidad de realizar las jornadas de evaluación y se procedió a repartir los cuestionarios a estudiantes y egresados, de manera que los puntos de discusión fueran precisamente los signos de crisis o problemas prioritarios identificados por los actores del proceso de gestión académico-administrativa de la EBA-LUZ (Pirela y Portillo, 2000).

Se logró recoger un total de 134 cuestionarios, los cuales se procesaron para definir las categorías identificadas por los informantes como áreas proble- mas. Una de esas áreas fue el "ambiente organizacional de la Escuela", en la que se definió como indicador la "implementación de modelos organizativos cónsonos con las actuales características de la universidad y la sociedad". En este sentido, se propuso asumir un modelo organizativo en el que se le otorgara importancia a la producción y comunicación del conocimiento, por tal motivo quedó en evidencia contar con una nueva forma de organizar la estructura académico-administrativa de la EBA-LUZ, caracterizada por la flexibilidad y la participación activa de los actores vinculados con la dinámica de gestión. Esta forma incluye la creación de $\mathrm{C}$ de $P$, que se conviertan en espacios para compartir y transferir el conocimiento.

\subsection{De los resultados del acercamiento empírico a la implementación de las estrategias medulares del enfoque $\mathbf{C}$ de $\mathbf{P}$}

El procesamiento de la información generada por los informantes y la consecuente formulación de la agenda de trabajo para las jornadas de evaluación, permitió abrir un espacio para la discusión acerca del nuevo modelo organizativo y la construcción colectiva de la filosofía de gestión de la EBA-LUZ, procesos que tiene que ver de manera directa con la primera y segunda estrategia de implementación del enfoque de $\mathrm{C}$ de $\mathrm{P}$, referida al redimensionamiento organizacional, pasando del modelo vertical, al horizontaldepartamentalizado.

La estructura organizativa, integrada por los departamentos de: Bibliotecología y Archivología, Gerencia de la Información e Investigación e Informática, sería apoyada por una Coordinación de Ex- 
tensión y una de Prácticas Profesionales, unidades desde las cuales se establecerían las relaciones con el sector externo.

En cuanto a la segunda estrategia, referida a la construcción colectiva de la filosofía de gestión, se expresó que la EBA-LUZ deberá: Ser una entidad científico académica de vanguardia en Ciencias de la Información, que integrada a las nuevas realidades y escenas proyectadas para la Educación Superior en el siglo XXI, proporcione el soporte docente $y$ de investigación requerido para la excelencia y competitividad, que permita visualizar el trabajo informacional de alto valor y consolidarse como ente consultivo del sector socioproductivo en el ámbito nacional e internacional.

En cuanto a la misión se decidió que la Escuela de Bibliotecología y Archivología de la Universidad del Zulia debe ser Una entidad científica académica social mente comprometida con la formación de profesionales integrales, responsables de la gestión de información y del conocimiento, a través de experiencias de aprendizajes, del desarrollo de investigación que generen alternativas del solución al sector socioproductivo de la región y el país.

No se trabajó con definición de valores, es una actividad que está pendiente, sobre todo si se quieren incorporar los principios operativos y funcionales de las comunidades de práctica. En este sentido, se proponen los valores de: confianza, colaboración e interdependencia.

En relación con la tercera estrategia medular, referida a la detección e identificación de las áreas temáticas alrededor de las cuales conformar las comunidades de práctica, se realizó una revi- sión de tendencias existentes en las universidades latinoamericanas y españolas que ofrecen programas o carreras de bibliotecología, archivología, documentación y ciencias de la información, con el propósito de determinar las áreas y líneas que manejan en universidades de México, Brasil, Cuba, Argentina, Venezuela, Puerto Rico y España (Ver Cuadro 1).

Luego de revisar las tendencias relacionadas con las orientaciones de los programas de postgrado, existentes en América Latina y España, y considerando el estudio realizado por Crespo et tal (2001), se cree pertinente que las comunidades de práctica que se deben conformar en la EBA-LUZ. son Análisis documental y sistemas de representación del conocimiento, Gerencia de la Información y el conocimiento y Estudios métricos de la información científica y tecnológica. Estas tres comunidades tomarían impulso y aliento en cada uno de los Departamentos que integran la Escuela: la comunidad análisis documental y sistemas de representación del conocimiento se conformaría por profesores del Departamento de Bibliotecología y Archivología; la comunidad de Gerencia de la Información y el Conocimiento por profesores del Departamento de Gerencia de la Información; y la Estudios Métricos de la información por el Departamento de Investigación e Informática (Ver Cuadro 2).

Se requiere entonces abordar el trabajo de nombrar a los líderes de la cada comunidad local, tomando en cuenta su trayectoria académica, para posteriormente comenzar a hacer los contactos con las universidades mencionadas y 


\section{Cuadro 1}

\section{Áreas y líneas prioritarias de desarrollo en el área de bibliotecología, archivología y ciencias de la información en América Latina y España}

\begin{tabular}{l} 
Países/Universidades \\
\hline Argentina \\
Universidad de Buenos Aires \\
Brasil \\
Universidad de Sao Paulo \\
Universidad de Brasilia
\end{tabular}

Cuba

Universidad de La Habana

México

Universidad Autónoma de México

Puerto Rico

Universidad de Puerto Rico

\section{España \\ Universidad Carlos III de Madrid}

\section{Áreas y líneas prioritarias de desarrollo}

Gestión de recursos de información

Diseño y análisis de sistemas de información

Acción cultural

Análisis documental

Generación y uso de la información

Información, desarrollo y sociedad

Tecnología de información

Análisis temático y descripción bibliográfica

Planeamiento y gerencia de unidades de información

Sistemas y redes de información documental

Estudios teóricos de las ciencias de la información

Organización y representación del conocimiento

Gestión de información

Diseño y elaboración de productos de información

Información, conocimiento y sociedad

Organización de la información documental

Tecnologías de la información

Sistemas y servicios bibliotecarios y de información

Usuarios de la información.

Conocimiento, desarrollo y sociedad

Diagnóstico para las necesidades de información

Industria de Información: Sus clientes y sus servicios

Organización y recuperación de la información

Tecnologías de información

Análisis de contenido

Gestión de documentos

Estudios del consumo de información científica

Problemas de organización del conocimiento

Recuperación de información electrónica

Fuente: Elaboración Propia.

comenzar a estructurar las comunidades de práctica a escala nacional e internacional.

Esta forma de concebir el conocimiento en la universidad permite, entre otras cosas, compartir experiencias enriquecedoras e intercambiar diversas visiones de los problemas que se investi- gan, con el propósito de generar alternativas de solución de manera colectiva.

\section{Consideraciones finales}

La propuesta formulada sobre la conformación de comunidades de práctica, en el contexto de un enfoque de gerencia del conocimiento para la Escuela 


\section{Cuadro 2 \\ Relación entre las Comunidades de Práctica, los Departamentos y los países participantes}

\begin{tabular}{lll} 
Comunidades de Práctica & \multicolumn{1}{c}{$\begin{array}{c}\text { Departamento responsable } \\
\text { de la EBA-LUZ }\end{array}$} & Países participantes \\
\hline $\begin{array}{l}\text { Análisis y representación } \\
\text { del conocimiento }\end{array}$ & $\begin{array}{c}\text { Bibliotecología y Archivología } \\
\text { Gerencia de la información } \\
\text { y el conocimiento }\end{array}$ & $\begin{array}{l}\text { España } \\
\text { Brasil } \\
\text { Puerto Rico }\end{array}$ \\
$\begin{array}{l}\text { Estudios métricos de la } \\
\text { información científica }\end{array}$ & Investigación e Informática de la información & Argentina \\
y tecnológica & & México \\
\hline
\end{tabular}

Fuente: Elaboración propia.

de Bibliotecología y Archivología de la Universidad del Zulia, permitió establecer relaciones entre los enfoques emergentes de las ciencias gerenciales, los cuales plantean la necesaria centralidad del conocimiento como un recurso estratégico de las organizaciones y los proyectos que actualmente se adelantan en la Universidad del Zulia, en relación con la transformación académico-administrativa.

El enfoque de $\mathrm{C}$ de $\mathrm{P}$, enmarcado en un contexto de gerencia del conocimiento, reconoce que el conocimiento acumulado por los miembros de la organización constituye un recurso esencial para impulsar el desarrollo la organización, por tal motivo la estructura debe girar en torno a la producción y transferencia del conocimiento, para lo cual se requiere concebir y desarrollar un modelo organizacional centrado en la comunicación, la relación y la reciprocidad. En este sentido, las propuestas organizacionales matriciales figuran como las más adecuadas a esta forma de trabajo.
La investigación gerencial empírica aporta elementos que indudablemente pueden ser utilizados como referentes para la formulación y reformulación de estrategias y para la definición de planes y programas que procuren mejoras continuas en la dinámica de gestión organizacional. La propuesta que se presenta en este trabajo es un ejemplo de ello.

Se recomienda entonces que los gerentes académicos de la EBA-LUZ asuman y ejecuten esta propuesta, que generaría alternativas de encuentro, debate y producción del conocimiento entre docentes-investigadores, egresados $y$ estudiantes.

\section{Bibliografía Citada}

Albornoz, O. (2000), Técnicas de la gerencia del conocimiento aplicadas a los espacios de producción del saber (Ps) en las empresas y en las instituciones de educación superior. Gerencia del conocimiento. Potenciando el capital intelectual para crear valor. Caracas: CIED-PDVSA. 
Arcila, F. (2000), Comunidades de práctica: Una alternativa de aprendizaje en y para las organizaciones. (Disponible en línea) En:http://www.gestiondelconocimiento.com/documentos2/ arcila/comunidades.htm.

Brown, J.S. (2000), Una visión distinta y prodigiosa. Revista Gestión. 2, 89-94.

Brown, J. S.; Collins, A.; Duguid, P. (1989), Situated cognition and culture of learning. Educational Researcher, 18, 32-42.

Consejo Universitario de la Universidad del Zulia. Comisión Organizadora de las Jornadas para la Transformación de LUZ. (S/F), Lineamientos teóricos, conceptuales y metodológicos para el proceso de conversión de los actuales departamentos de las Escuelas de LUZ, en Departamentos de las Facultades. Maracaibo: Universidad del Zulia.

Davis, K. y Newstrom, J. W. (1991), Comportamiento humano en el trabajo: Comportamiento organizacional. México, Mc. Graw Hill.

De Galicia, F. y Castillo, E. (2000), La educación superior en América Latina: Instituciones públicas y privadas. (Disponible en línea): http://www.sadpro. ucv.ve/agenda/. Última consulta: 10 de julio de 2002.

Diccionario de la Lengua Española (1992), Real Academia Española. Madrid.

Dirección General de Planificación Universitaria. (1998), Plan de desarrollo estratégico. DGPU. Universidad del Zulia.

Figueras, C. (1998), Tendencias y perspectivas de los estudios de Postgrado en Ciencias de la Información en Iberoamérica y el Caribe. (Foro) En: Memorias del IV Encuentro de Educadores e Investigadores de Bibliotecología, Archivología y Ciencia de la Información de Iberoamérica y el Caribe. Maracaibo: Universidad del Zulia.
Goldman, A. (1998), The Knowledge in a Social World. Oxford Universty Press.

Hacking, I (1996), Representar e intervenir. Paidos: Barcelona.

Mc. Dermott, R. (2000), ¿Cómo asegurar el éxito de las comunidades de conocimiento? Gerencia del conocimiento. Potenciando el capital intelectual para crear valor. Caracas: CIED-PDVSA.

Pasquali, A. (1998), Bienvenido global village. Caracas: Monte Ávila Editores.

Pirela M. J. y Portillo, L. (2000), Redimensionamiento organizacional de la Escuela de Bibliotecología y Archivología de la Universidad del Zulia: Una experiencia de evaluación institucional. Ponencia presentada en el $\checkmark$ Encuentro de Educadores e Investigadores de Bibliotecología, Archivología y Ciencias de la Información de Iberoamérica y el Caribe. GranadaEspaña: Universidad Euro Árabe.

Sánchez, S. (2000), Nuevas transfiguraciones de la información: La gerencia del conocimiento. Conferencia dictada en el $\checkmark$ Coloquio de Tecnologías Aplicadas a los Servicios de Información. Barquisimeto: Universidad Centro Occidental Lisandro Alvarado. Julio.

UNESCO (1998), Declaración Mundial sobre la Educación Superior en el Siglo XXI: Visión y Acción. Conferencia Mundial sobre la Educación Superior. [En línea]: http://www.rau.edu.uy/rau/docs/ parís1.htm>. [Consulta: 21 septiembre 2001].

Wagensberg, J (1994), Ideas sobre la complejidad del mundo. Barcelona (España): Tusquet editores.

Wah, L. (1999), Mucho más que una moda. Revista Gestión. 2 63-71.

Wertsch, J (1985), Vigotsky y la formación social de la mente. Barcelona: Paidós. 